\title{
Aboriginal Participation in Health Service Delivery: Coordinated Care Trials in the Northern Territory of Australia.
}

\author{
INTERNATIONAL JOURNAL OF SERVICES TECHNOLOGY AND \\ MANAGEMENT, 2003: 5, 45-62.
}

Gary Robinson, Peter d'Abbs, Ross Bailie, Samantha Togni.

\begin{abstract}
Australian Aborigines have much higher rates of illness and life expectancy up to twenty years lower than the Australian population as a whole. Community based primary health care to Aborigines in the Northern Territory of Australia has historically been under-resourced in part due to distinctive characteristics of the Australian health care funding system which has disadvantaged rural and remote regions. From 1998, the Northern Territory was the site of two Coordinated Care Trials (CCTs) which sought to achieve three main objectives: to significantly increase funding available to health services; to implement a system of clinical best practice; and to improve Aboriginal participation in health service delivery through the establishment of Aboriginal community Health Boards to act as funds managers and providers of health services to the trial populations. The paper outlines general CCT outcomes according to the findings of the commissioned evaluation studies, and examines the possibilities and constraints encountered in improving Aboriginal participation in complex health service developments.
\end{abstract}

\section{Key Words}

Australian Aboriginal health; coordinated care trials; health service evaluation; funds pool management and area health boards; Aboriginal participation and community control of health services; chronic diseases; care plans and care coordination; population health care.

\section{Introduction.}

Improving the health of Aborigines is a priority of Australian governments at the state and Commonwealth levels. Aboriginal life expectancy remains well below that of other Australians, and is lowest in the Northern Territory, a sparsely populated, tropical and semi-tropical region occupying one-sixth of the Australian landmass, but containing approximately 187,000 people, of whom $28.5 \%$ were of indigenous descent in 1999 (McLennan \& Madden, 1999). Aborigines suffer extremely high rates of chronic illness, including renal disease, diabetes and cardio-vascular disease, while infant mortality rates in the Northern Territory currently persist at over twice the non-indigenous rate (ibid.: 88 , 92ff.)

Australians generally enjoy a high standard of health care through universal public health insurance (the Medical Benefits Scheme, MBS) which secures access to primary health care delivered by general practitioners, and to subsidized pharmaceuticals through the Pharmaceutical Benefits Scheme, (PBS). However, particularly in rural and remote regions, Aboriginal access to primary health services funded through these schemes is low compared with other Australians, largely because of the absence of general practitioners and pharmacies (McDermott, 1995). In the Northern Territory (NT), most services in these regions are provided through community health centres funded by the Northern Territory Government's Territory Health Services (THS). They are usually 
staffed by Registered Nurses (RNs) and Aboriginal Health Workers (AHWs) recruited from the local communities. Salaried departmental general practitioners known as District Medical Officers (DMOs) make regular one to two day visits to the communities. The Northern Territory Government has long been concerned at the lack of funding of health care in the regions through the Commonwealth MBS and PBS schemes, and at the limits to its ability to expand health services without additional Commonwealth support.

In an attempt to overcome these problems, the Commonwealth Government in 1996 agreed to sponsor a number of Aboriginal Coordinated Care Trials (CCTs). In the NT, the CCTs saw a significant increase in funding for services, the establishment of integrated regional systems of service delivery where only disparate services had existed before, the introduction of computerized infrastructure supporting electronic medical records and care plans, and the establishment of Aboriginal Health Boards as fundholders and purchasers of services provided to trial populations. Four Aboriginal trials were established: two in the NT, one in Western New South Wales, and one in Western Australia ${ }^{1}$. The four trials were comprehensively evaluated at the local and national levels.

\section{Aims}

This paper draws on the authors' research as members of the two teams responsible for the local evaluation of the NT Coordinated Care Trials. The full range of issues relating to Aboriginal participation, community control and community development and concerning the Health Boards' roles as Aboriginal decision-making bodies cannot be considered here. The present paper primarily focuses on Aboriginal participation in clinical care.

We begin by describing the trials and the contexts in which they occurred, outlining the methods of the evaluation projects. We then set out the contributions to Aboriginal participation and community development anticipated by the logic of health service reform within the CCTs. We examine the specific changes to clinical practice introduced by the trials, and the extent to which these changes contributed to expected outcomes: improved clinical services and increased participation by consumers in their own health care. While improved health outcomes were the ultimate rationale for the CCT, these were not thought to be attainable within the two year trial period. Focusing on care coordination, we go on to explore the complexity of issues underlying Aboriginal participation in the development and delivery of clinical services - mainly through the involvement of locally recruited Aboriginal Health Workers.

\footnotetext{
${ }^{1}$ See Commonwealth Department of Health and Aged Care, March 2001, "The Aboriginal and Torres Strait Islander Coordinated Care Trials: National Evaluation Summary", for an outline of the four Aboriginal Coordinated Care Trials. The New South Wales and Western Australian trials differed substantially from the two NT trials.
} 


\section{Regional Context and Duration of the Northern Territory Trials.}

Between 1997/8 and 2000, two Aboriginal Coordinated Care Trials (CCTs) were established in remote areas of the NT, one on Bathurst and Melville Islands (collectively known as the Tiwi Islands) near the capital city, Darwin, and the other in the Katherine West region, an area covering 162,000 sq.km between the town of Katherine and the Western Australian border. The Tiwi people, numbering just over 2,000 persons, are traditional owners of Bathurst and Melville Islands under the Commonwealth Aboriginal Land Rights Act, Northern Territory (1976) The population is largely homogeneous, speaking both Tiwi and English languages, with a small number of non-Tiwi employed in service delivery and governance in the Local Community Government Councils of the three main communities. The Katherine West region contains some 3,000 people, of whom $85 \%$ are Aboriginal. Today, the linguistically disparate indigenous population of the region has regained title to much, though not all, of the land in or around the eight remote communities of the trial region. Most of the non-indigenous population in the region lives on cattle stations or in the small town of Timber Creek.

The 'live phase' of the Tiwi CCT commenced in December 1997, that of the KW CCT in July 1998. The 'live phase' of both trials ended on 31 December 2000, and was followed by a 12 month 'transition phase', during which funding and other arrangements introduced in the Trials were maintained while the Commonwealth Government considered the findings of evaluations and resolved its future commitments. At the time of writing, the institutional and financial arrangements of the CCTs remained in place in both Trial sites.

\section{Objectives of the NT Coordinated Care Trials}

Implicit in the 'program logic' (McClintock, 1990) underlying CCT objectives was a proposition that three major reforms, acting together, would lead in the short term to improvements in delivery of health services which would, in due course, bring about better health outcomes. The anticipated contributions of the three main program components can be summarised as follows:

1. Establishment of regional Health Boards consisting of predominantly elected all-Aboriginal memberships drawn from the participating communities, would lead to enhanced community control of health services in the trial regions;

2. Additional funds available within a funds pool would encourage reform of existing services and introduction of new health services and programs, in particular community-based preventative programs;

3. Care coordination would lead to (a) more systematic and timely delivery of services and (b) enhanced participation by consumers in decisions relating to their own health care, which together would lead in turn to (c) more effective clinical prevention and management of existing conditions.

The effects of Aboriginal participation were expected to be felt within each of these program components and were supported in the course of trial development processes through attention to recruitment of AHWs and Aboriginal Community Workers, and 
through the development of training and management strategies involving Board members and other indigenous personnel.

\section{Evaluation Methods.}

Following Pawson's and Tilley's (1997) model of 'realistic evaluation', the capacity of each of the three main interventions to act as change mechanisms and thus to produce specific outcomes was defined by their interaction with influences in the trial context, that is, in predominantly indigenous communities and cultures into which modern medicine has only relatively recently been grafted. Both evaluation teams sought to assess the response of Aboriginal and non-Aboriginal actors to these interventions, in order to grasp the degree to which they became effective change mechanisms within the CCT. Each of the three interventions implied action relating to Aboriginal participation and community development. Each was consequently constrained by characteristics of the pre-trial situation in terms of the existing capacity of the system to achieve Aboriginal involvement in key processes. However, the processes of Aboriginal participation and involvement, and their presumed flow-through to improved service delivery and possible improvements in wellbeing were the least well conceptualized within the trial objectives.

The local evaluations took place under the broad general direction of a National Evaluation Framework which focused on direct and 'intermediate' indicators of health gain resulting from care coordination and paid little attention to the considerable social, political and managerial complexity of the task of creating new regional service organizations under Aboriginal Boards.

The local evaluators developed their own approaches to this element of the CCT projects. There was variation in the approaches taken by the two evaluation teams to analysis of decision-making and management by the Boards, and to interpretation of the effectiveness of Aboriginal participation in service delivery as each team responded to specific local conditions of each trial and the circumstances of dialogue with their respective Boards. In general, the evolution of the Health Boards' roles as purchasers and providers of health services, of decision-making by the Boards, and of the Boards' relationships with other stakeholders were monitored through attendance at meetings, semi-structured interviews with Board members and others, and investigation of documentary sources. In the process, each team developed its own pattern of dialogue with the respective Boards and stakeholders concerning Health Board decision-making and strategies for community participation and control.

By comparison, the evaluation of care coordination was more heavily prescribed by specific technical innovations. The basis of care coordination was the computerised information system, known as the Coordinated Care Trial Information System (CCTIS), developed and implemented by THS with some Commonwealth financial assistance. This contains electronic medical records for all clients. Consultation areas in each health centre have a computer terminal linked to a local server, which contains the local database enabling search of records, creation of lists of clients due for recall or of services due, reports of results, and so on. Data is regularly transferred to a central database maintained by THS, where it is merged with hospital and other data for 
aggregate analysis of services, results, hospitalization, morbidity, and other factors. The best practice guidelines developed by the THS team are loaded in CCTIS as care plans which are assigned to all clients. The care plans are of two kinds. Population care plans assign routine monitoring and other preventive services and screening in the age categories 0-3 years, 4-15 years, 16-49 years, and 50+ years. At the commencement of the Trials, all persons were automatically assigned the appropriate population care plan. The second kind of plan, standard disease specific care plans, represent practice protocols for care and management of common serious conditions including hypertension, diabetes, renal disease, rheumatic heart disease, chronic obstructive airways disease, and for chronic ear disease and failure to thrive in infants and children. Practitioners combine population and standard care plans to produce personalized care plans for individuals according to health problems present, as part of each individual client's record in CCTIS.

In both evaluation projects, care coordination was evaluated by measurement of change in services delivered to correspond with care plan specifications, in other words, measurement of the shift in the mix of services delivered to reflect the adopted best practice model. This was achieved through audits of patients' medical files. A stratified random sample of health centre clients' files was manually audited against the protocols for population care plans at baseline in late 1998, in mid 1999, at the end of 1999 and again a year later at the end of 2000. The sample was stratified to include numbers for each of the population care plan age groups, and for each of the main health centres. A random sample of diabetics was also audited at the same intervals against the protocol for the diabetes care plan. Because the entry of data in CCTIS was assumed not to be complete, the audits examined all systems of client records: in CCTIS, and all locations in the patients' paper file. A specified service was considered to have been carried out if it was recorded as having been delivered in any or all of these records. This procedure also enabled the audits to gather comparative data on the levels of entry of all service items in CCTIS and the existing systems of records over the four audits. It was assumed by CCT management and researchers that accountability in delivery of services, the ability to work to protocol and to set objectives for services to particular client groups, could only be established through systematic recording of services delivered, and that improvements in recording of services were in themselves an indicator of potential for improved coordination of care $^{2}$.

In addition, changes in clinical practices and in relationships between health providers and consumers were monitored by observation and interviews with attention given to human and material resources in the health centres and to patterns of use of the computer system and the organization of consultation activity by practitioners. In the Tiwi CCT, circumstances allowed for comparative analysis of data on consultations entered by Aboriginal and non-Aboriginal practitioners in CCTIS.

\section{Aboriginal Participation in the Trials}

\footnotetext{
${ }^{2}$ A small number of biochemical indicators (blood pressure, blood sugar, $\mathrm{HbAlc}$ ) was also collected for the diabetic sample. However, it was not expected that measurable gains in health status would occur within the timeframe of the CCT. An analysis of the relevant findings is forthcoming.
} 
The development of Aboriginal participation and the development of community capacity and infrastructure can be traced for the three key interventions of the coordinated care trials. Figure 1 sets out the changes from the pre-trial situation at left, to the right side of each horizontal row.

Figure 1: Aboriginal participation and key structural reforms of the CCT

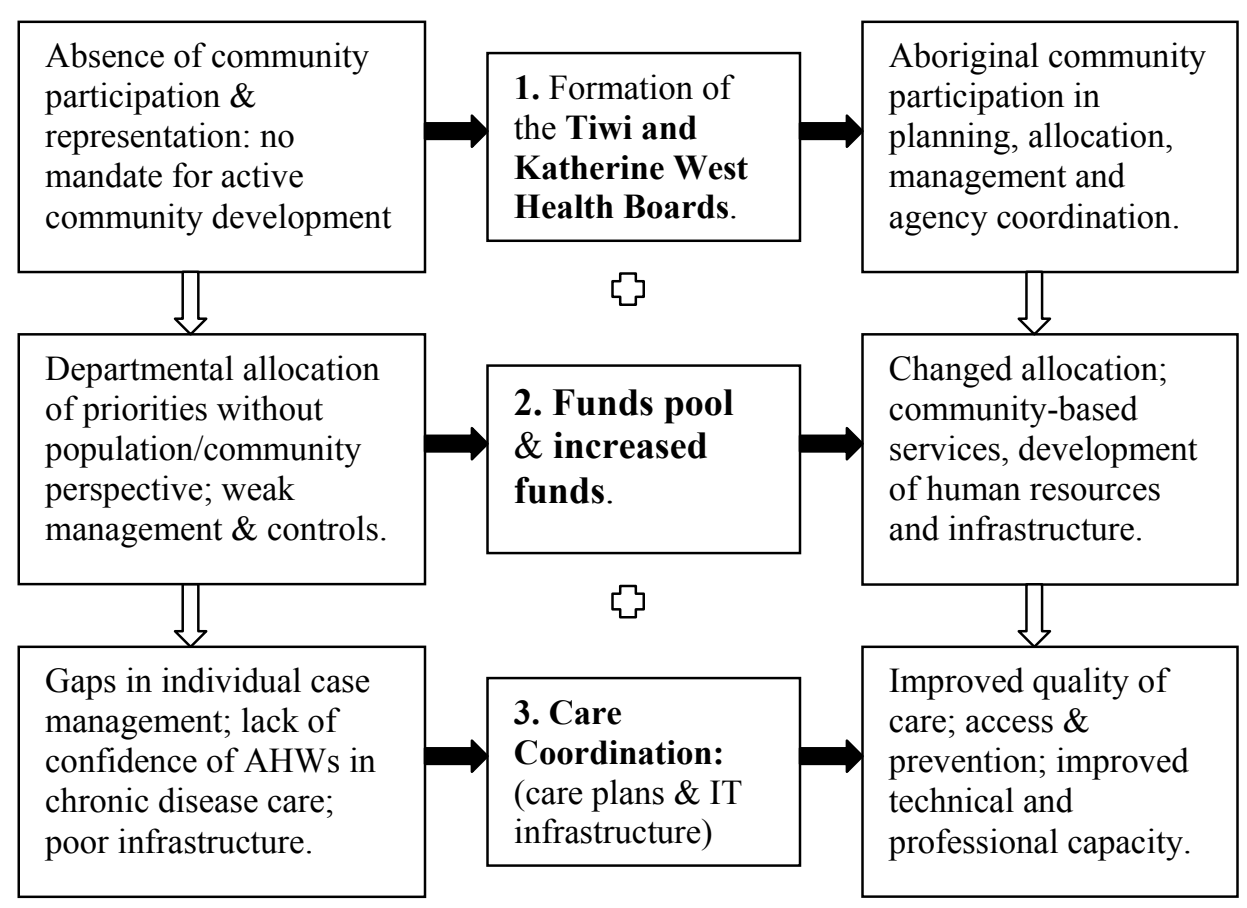

\subsection{Community participation and control through the Boards}

In the pre-trial period, the establishment of the Health Boards involved detailed consultation with Aboriginal stakeholders to gain support for the trials and then to establish the Boards, drawing on existing mechanisms for Aboriginal participation. In the Tiwi case, this was actively supported by the Tiwi Land Council, an already existing Aboriginal decision-making body which had established the Tiwi Health Board before the formation of the CCT. The Land Council saw the CCT as an opportunity to assert local Aboriginal leadership concerning serious health issues. In the case of Katherine West, the Trial region did not so clearly coincide with existing political structures, and the formation of the KW Health Board involved negotiations through the relevant regional structures of the Aboriginal and Torres Strait Islander Commission (ATSIC, the Commonwealth agency responsible for administration of Aboriginal affairs) with initial organizational support and assistance of an Aboriginal Medical Service (AMS) located in Katherine, the nearest major population centre. Despite the differences, in both cases, the establishment of the CCTs and the Health Boards drew Aboriginal community 
representatives into extensive consultative processes and established potentials for Aboriginal involvement in high level decision-making in health service delivery of a kind unknown within previous departmental arrangements. It created Aboriginal organizations with legitimacy and resources sufficient to enable them to mobilize collaboration between community agencies and institutions (schools, local councils, housing associations, etc.) on matters of priority, as well as to engage with the many other external agencies and departments which provide services within the communities.

\subsection{Funds pool and "cash-out".}

The capacity of the Health Boards to introduce change was enhanced by the injection of additional funds. These took the form of a per capita, per annum 'cashout' of MBS and PBS monies at a rate equivalent to the average amount expended through those schemes by Australians nationally - \$AU536 per capita in 1997 - paid to each Board on the basis of their respective estimated trial populations. These funds were added to funds already allocated by THS to health services in the trial regions to form a total pool of funds for services for each trial. The total funds, including further funding to support the costs of administration by the Boards, resulted in an approximate doubling of expenditure on services compared with the pre-trial situation. While the health boards were obliged to ensure provision of basic clinical services, it was expected that the mechanism of the funds pool would enable them to develop their own priorities and new programs.

The pooling of Commonwealth and Northern Territory funds for all services created for the first time a basis for management of service delivery according to regional and community perspectives. Management had previously been applied weakly, health centre by health centre, with little broad coordination of effort or strategy. The CCT created stronger demands for accountability in service delivery than had existed within the old departmental regime. Many programs had been the product of single practitioner initiatives within single communities, with little overall coordination, and usually unsupported by adequate information and evaluation. Other departmental programs were constrained by the limitations of local infrastructure, available human resources, and the limited ability of practitioners to mobilize support in the communities among local councils and community members.

Among the evidence for the development of Health Board decision-making through the mechanism of the funds pool, is the fact that both Boards actively moved beyond the role of funds pool manager and purchaser of services (from THS and other providers), to become the major providers of health and community services to their regions. All health centres but one were under the management of THS at trial commencement. The Tiwi Health Board was provider-manager at the largest centre, Nguiu, from the beginning of the CCT, and took over full management of the other two health centres from THS in April 1999. Katherine West took over management of four health centres in October 1999 (that is, just before the end of the live phase of the trial), with two further health centres taken over in September 2000, (towards the end of the transition phase), while two remain under THS management. Both Boards have extended services beyond primary health care into a range of preventive and health-promotional strategies. With the flexibility provided by the funds pool and the increased funds, the Boards have sought to tackle environmental health and other areas of community public health, including 
mental health and suicide prevention. These developments have produced rapid growth of Aboriginal employment in community based services and in turn have demanded concentrated attention to development of human resources, specifically to training and support for higher levels of competence and responsibility among Aboriginal employees. Given education levels and limited experience within the communities, this is necessarily a medium to long term objective.

\subsection{Care coordination}

The third major intervention, the implementation of care coordination, was based on best practice protocols in the form of electronic care plans to guide clinical service delivery, and on use of CCTIS to manage proactive preventive delivery of services to various groups of client according to health problem, age and gender. THS conducted a thorough developmental process, reviewing practice across the NT, and national and international literature, and included clinicians, doctors, nurses and a senior Aboriginal Health Worker on the development team. It was intended to achieve the systematic delivery of population-wide preventive clinical measures, and above all, to improve prevention and treatment in response to the serious levels of chronic illness in the communities. It aimed to support a significant improvement in professional capacity of all personnel including Aboriginal staff in the health centres. Training and support for use of the computerized system and care plans was provided by THS throughout the live phase of the CCT; this is now largely the responsibility of the Boards.

Training and development for care coordination focused on use of CCTIS to enter care plan services and results of measures and tests, and to a lesser extent on its use as the primary database for patient recall. The implementation strategy of itself did not address the complexities of local health centre management, in terms of interdisciplinary relationships between Aboriginal and non-Aboriginal clinicians, management of case load through both opportunistic delivery of services and planned recall of groups of persons with particular problems or particular kinds of services due. Furthermore, basic tasks of data entry proved to be influenced by specific local circumstances, including variable staff competencies, limited access to computer terminals and inadequacy of physical facilities, and a range of factors to do with the pre-existing organization of work practices and the style of management of case load. In short, at the micro-level, implementation of care coordination was left to evolve under the Boards' direction, with the result that for many reasons, management of clinical practices remained to some degree fluid and unsettled throughout the CCT, with different local strategies evolving within the two CCTs and in their various health centres.

\section{Results}

The primary question to be answered by the clinical audits was:

a. To what extent were services delivered as scheduled by the designated care plans (population care plans and diabetes care plans)?

Supplementary questions investigated through observation and interview included: 
b. For the Tiwi CCT, what were the respective roles of Aboriginal and nonAboriginal clinicians in service delivery and in particular their respective contributions to any recorded change in services delivered?

c. To what extent did changes in clinical practice lead to increased participation by Aboriginal consumers in their own health care?

\subsection{Changes in service delivery}

Tables 1 and 2 set out the changes in the pattern of services delivered, as a measure of the proportion of persons who received service items prescribed by their care plans at each audit.

Table 1: Proportion of services delivered as required by care plan, $\mathrm{KW} \mathrm{CCT}$

\begin{tabular}{|c|c|c|c|c|c|}
\hline Care Plan group & $\begin{array}{c}\text { Baseline } \\
\text { Nov } 1998\end{array}$ & $\begin{array}{l}\text { Mid-term } \\
\text { May } 1999\end{array}$ & $\begin{array}{c}\text { Final } \\
\text { Nov } 1999\end{array}$ & $\begin{array}{l}\text { Transition } \\
\text { Nov } 2000\end{array}$ & $\begin{array}{l}\text { Chi square for } \\
\text { linear trend } \\
\text { p values }\end{array}$ \\
\hline $0-3$ years & $97 / 21645 \%$ & $89 / 21442 \%$ & $103 / 17858 \%$ & $55 / 10353 \%$ & 0.462 \\
\hline $4-15$ years & $70 / 12357 \%$ & $60 / 11055 \%$ & $52 / 9257 \%$ & $53 / 8265 \%$ & 0.716 \\
\hline $16-49$ years & $84 / 32826 \%$ & $105 / 31633 \%$ & $134 / 30544 \%$ & $98 / 34029 \%$ & 0.672 \\
\hline $50+$ years & $134 / 38935 \%$ & $146 / 39037 \%$ & $172 / 39144 \%$ & $167 / 35148 \%$ & 0.386 \\
\hline Diabetes sample & $684 / 203034 \%$ & $701 / 203035 \%$ & $825 / 207040 \%$ & $508 / 186027 \%$ & 0.611 \\
\hline
\end{tabular}

Table 2:Proportion of services delivered as required by care plan, Tiwi $\mathrm{CCT}^{4}$

\begin{tabular}{|c|c|c|c|c|c|}
\hline Care Plan group & $\begin{array}{c}\text { Baseline } \\
\text { Nov } 1998\end{array}$ & $\begin{array}{l}\text { Mid-term } \\
\text { May } 1999\end{array}$ & $\begin{array}{c}\text { Final } \\
\text { Nov } 1999\end{array}$ & $\begin{array}{l}\text { Transition } \\
\text { Nov } 2000\end{array}$ & $\begin{array}{l}\text { Chi square for } \\
\text { linear trend } \\
\text { p values }\end{array}$ \\
\hline $0-3$ years & $122 / 27844 \%$ & $114 / 21653 \%$ & $130 / 18969 \%$ & $57 / 7873 \%$ & 0.138 \\
\hline $4-15$ years & $58 / 11053 \%$ & $31 / 12026 \%$ & $89 / 11081 \%$ & $105 / 13976 \%$ & 0.052 \\
\hline $16-49$ years & $317 / 64749 \%$ & $317 / 65848 \%$ & $373 / 63659 \%$ & $402 / 66960 \%$ & 0.112 \\
\hline $50+$ years & $191 / 42045 \%$ & $207 / 52249 \%$ & $257 / 41063 \%$ & $239 / 36466 \%$ & 0.119 \\
\hline Diabetes sample & $843 / 194343 \%$ & $862 / 194344 \%$ & $1111 / 185660 \%$ & $1133 / 183062 \%$ & 0.010 \\
\hline
\end{tabular}

In both CCTs, audits conducted at baseline and six months later (in May 1999) indicated that there had been little apparent change in the overall level of services delivered according to protocol for most groups. By the end of 1999, however, for the Tiwi CCT, an increase (observed, but not statistically significant) in the delivery of services according to protocol for all care plans - including both the population care plans and the diabetes care plan - was evident. During the final "transition" year to December 2000, these improvements were sustained for the Tiwi Trial, with continuing improvement in delivery of services in some areas, and a leveling out in others. In Katherine West, the

\footnotetext{
${ }^{3}$ Source: D'Abbs, P., et al., 2001. Chi squares calculated on average services scheduled and delivered for each sample.

${ }^{4}$ Source: Robinson, et al., 2001: Part 2, Clinical Services and Care Coordination, tables $19-23$. Chi squares calculated on average services scheduled and delivered for each sample.
} 
picture was much more variable across the various sites audited, with no statistically significant increases in services delivered overall, but observed improvement in some individual health centers.

At the mid-term audits for Tiwi and Katherine West, there were significant increases in the recording of services in CCTIS. For example, the Katherine West six-month audit of electronic and paper records showed that only $35 \%$ of specified services for diabetics had apparently been provided, compared with $34 \%$ at baseline; however, of the services provided, $49 \%$ had been recorded in CCTIS at six months, compared with $35 \%$ at baseline (Local Evaluation Team, 2000: Sections 20.1 \& 21.1). For Tiwi, the improvements in recording were from $50 \%$ for all care plans at baseline, to $67 \%$ at the final audit in December 1999 (Robinson, et al., 2001; Robinson \& Bailie, 2000). That the increase in recording of services in CCTIS increased before improvements in delivery of services according to protocol suggests something like the following simplified sequence:

Figure 2: CCTIS and general sequence of changes in service delivery

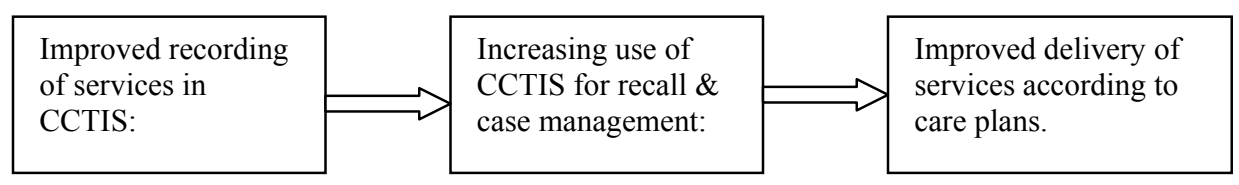

As general levels of data entry improved, CCTIS was increasingly used by practitioners as the primary recall instrument, for example to systematically deliver population plan checkups such as pap smears or vaccinations, as well as checkups for persons with health problems such as diabetes. This in turn contributed to improved opportunistic delivery of care plan services overall as suggested by the sequence in Figure 2. In both CCTs, there was great variation in the extent to which specific service items were provided and/or recorded in CCTIS. For example, whereas monitoring of blood pressure of diabetics was carried out at required intervals in at least $80 \%$ of cases in the Katherine West samples, a number of other services were delivered at less than $33 \%$ of recommended levels in all four audits, namely body mass index, waist circumference, eye examination for cataracts, feet examinations for sensation, reflexes, pressure areas and infections, laboratory investigation of fasting lipids and counselling/advice on exercise, weight loss, smoking and alcohol.

Services for which there are already strong emphases on recording of precise values (such as blood pressure, weights, delivery of vaccinations to infants) were in general recorded at high levels in CCTIS (80 - 100\%). Other care plan services, such as health promotional counseling on lifestyle, were initially very poorly recorded in CCTIS, and better recorded in paper notes. For the Tiwi CCT, the level of entry of these services in CCTIS improved significantly during 1999 in response to feedback from evaluators, but again declined in 2000 .

Despite the differences in staffing and in achieved service levels in the two trials, attendance levels for most groups of clients were high, and brought into focus a key 
problem for the ongoing development of care coordination. High attendance levels - for example over $80 \%$ of diabetics in KW and over $90 \%$ of Tiwi diabetics sampled had visited the health centre within three months of the audit - pointed to opportunities for far higher levels of opportunistic delivery of care plan services than had been achieved during the CCTs, even where improvements in delivery had been observed. This represents a complex challenge for practitioners and management alike.

The difference in outcomes between the two trials as recorded in tables 1 and 2 deserves some comment. The live phase of the KW CCT commenced six months later than the Tiwi CCT. Moreover, the transfer of management of THS-managed health centres to management by KWHB occurred much later than in the case of the Tiwi, as noted. Perhaps more importantly, the Tiwi Islands had been the site of a number of major research projects during the 1990s. These included a comprehensive screening and treatment program for renal disease and ear treatment programs for babies and children. Notwithstanding many deficiencies, it is probably fair to say that Tiwi health centres partly through closeness to Darwin, partly through research - have been able to sustain resources at levels somewhat higher than some of the remoter regions of the Territory, including Katherine West, where there have been greater difficulties building clinical capacity through recruitment, training and retention of AHWs, and retention of nonAboriginal staff. These differences between the Tiwi Islands and Katherine West were evident in generally higher levels of diagnosed health problems among the Tiwi, higher clinic attendance levels for some patient categories, including diabetics, and higher or at least more consistently sustained levels of Aboriginal staffing.

\section{Changes in Clinical Practice}

In both CCTs, the implementation of CCTIS was marked by instances of practitioner resistance - to the point at which, for example, the Tiwi Health Board did not renew the services of some medical personnel and sought to implement in employee agreements strong clauses on use of the computer system. Other practitioners found difficulty with the new standards of accountability based on the care plans and information system. Many remote area health personnel are used to working idiosyncratically and are frequently subject to only limited managerial intervention and demands for accountability; many do not think in organizational, 'strategic' or 'population' terms in relation to practice objectives. Perhaps more importantly, they operate in contexts in which relations between Aboriginal and non-Aboriginal practitioners produce their own constraints on practice objectives.

In Katherine West, one DMO who resigned during the live phase prepared a written critique of CCTIS in which she remarked that she felt as if she were "serving the system rather than it being a tool for patient management". A DMO in the Tiwi CCT was open in campaigning against CCTIS and in resisting its use. An experienced RN in Katherine West commented that the CCT had revealed just how inadequately the health centres were resourced to provide best practice in the care of clients. In her view there was still a long way to go to achieve an effective level of care planning and therefore care coordination; there was still, she asserted, a "herding mentality... we round them up, 
bring them in, prod and poke them, send them back.... We're [the health centre staff] still taking responsibility.... It hasn't shifted to helping family care for that individual." (Local Evaluation Team 2,000: 69)

In fact, such practitioner angst about the effectiveness of the reforms, while sometimes pointing to systemic problems, usually also expressed a discomfort with the demands of managerial pressure to increase both opportunistic and planned delivery of care plan services, rather than accurately representing the experience of patients in whose name the concerns were often voiced. This became an implicit commentary both on the difficulties of establishing new and effective management processes in the health centres, and, as the comments of the $\mathrm{RN}$ indicate, on unresolved questions about how best to engage their Aboriginal clients.

Perhaps the most important factor shaping community involvement in primary health care services is the role of AHWs in service provision. Most AHWs are locally recruited from the communities and have predominantly on-the job training, with variable levels of literacy and numeracy. Some pass through certificate level vocational training away from the communities at an indigenous training institute. The relationship between AHWs and other practitioners is at once an interdisciplinary relationship and a cross-cultural one. While there is relatively high turnover among the comparatively highly trained nonAboriginal staff, among whom only a minority stay in one location for more than two years, Aboriginal Health Workers are community members, many of whom have worked in their positions for ten years or more. They are often very adept at managing community input into health activities, at consultation with community members and at facilitating decision-making in which community consent is at issue. The continuity they represent is offset by the frequent renegotiation of roles with new nurses and doctors, many of whom are highly motivated to achieve change, while others are merely idiosyncratic in their preferences. Before the CCT, these renegotiations of practice occurred within a weak overarching structure of management and coordination by THS.

In general, at both locations, AHWs appear to have strongly embraced the idea of Aboriginal ownership of the services through the Boards. However, the development of their roles in service delivery has been complex, and their responses to the challenge of care coordination are an important measure of some aspects of the reform agenda. In Katherine West, the numbers of AHWs working in the system had fallen substantially before the CCT. THS had previously delayed replacement of (Aboriginal and nonAboriginal) staff as a means of stretching limited budgets, and in some health centres, some positions had remained unfilled for some time. For much of the CCT, the main objective of the Katherine West Health Board was to establish continuity in professional capacity in the health centres at a level required to support the program, with training, recruitment and retention of AHWs (and resident GPs to replace the DMOs) foremost.

In the Tiwi health centres, relatively more developed participation by AHWs and more reliable attribution of consultation information in CCTIS to practitioner categories, enabled evaluators to monitor the AHW contribution to changes in recording and delivery of care plan services. Assessments conducted by THS before the CCT had indicated a 
lack of confidence on the part of AHWs with aspects of medical management of the chronically ill; this was in part to be addressed by the new infrastructure and protocols. However, examination of the audit findings and other data in CCTIS, in combination with observation and interviews, shows the contribution of AHWs to the improvement in the pattern of services noted in Table 2 to be ambiguous. The proficiency of AHWs in use of CCTIS varies greatly, with some very competent users, and others who use the system infrequently, if at all. This variation also existed across the care plans, with some areas of care plan activity showing higher AHW involvement and recording high levels of use of the system by the AHWs, approaching that of any other practitioners.

In general, improvements in use of CCTIS by Tiwi AHWs recorded during 1999 appear not to have been sustained in 2000, after initial achievement of a basic proficiency with entry of records in CCTIS. Throughout the CCT, Tiwi AHWs entered some $57 \%$ of all consultations in CCTIS, with nurses entering just under $30 \%$. However, of care plan service items (in chronic disease care plans) delivered during consultations, AHWs entered just under $20 \%$, while nurses entered over $60 \%$ (Robinson et al, 2001: 52$)^{5}$. In other words, AHWs appear to have remained oriented to delivery of acute and ad hoc types of services. It is thus clear that much of the change in the mix of services delivered during the CCT was achieved by the non-Tiwi personnel, supported by AHWs, albeit in ways which are difficult to assess.

There appear to be strong continuities in relations between Tiwi AHWs and their Tiwi clients, which undoubtedly to some extent underlie the persistence of orientation to reactive, acute care styles of delivery, and which in turn shape possibilities of change in process and strategy in clinical practice. That is, the capacity of the Tiwi AHWs to respond to changes introduced by the CCT was constrained by existing relationships which include relations between AHWs and Tiwi clients and between AHWs and nonTiwi medical staff. The latter are characterized by a tendency to allow outsiders to renegotiate aspects of the professional terrain provided the changes do not disrupt the basic relationships within which AHWs understand their role within the communities.

The evaluation has therefore highlighted an important challenge for the Health Boards which extends beyond the problem of securing Aboriginal participation through recruitment of AHWs. There is a need to better understand the orientation of AHWs, the constraints on their capacity to use the new system and the potentials for them to take higher levels of responsibility for clinical care through use of CCTIS and care plans without devaluing their current role and status within the system. Neither the existence of the Health Boards - without development of explicit strategy - nor the implementation of the technical instruments of care coordination are by themselves sufficient to achieve these improvements.

\footnotetext{
${ }^{5}$ CCTIS records services delivered as part of a care plan as a category separate from acute and ad hoc items of service.
} 


\section{Consumer Participation in Health Care}

One of the expectations of the CCTs at the outset was that care coordination would involve not only the use of best practice clinical guidelines, but also consultative clinical processes in which clinicians would engage with patients and involve them in the formulation of personal care plans. Through these processes, patients would be "empowered" by participation in planning their own health care.

Care planning in the context of the CCT is a three stage process:

1. Automatic assignment of a population care plan to every resident registered on CCTIS;

2. Assignment of personal care plans based on relevant population care plan and diseasespecific standard care plans;

3. Monitoring and management of patients according to the schedules in the personal care plans.

In Katherine West, the expectation of patient participation generated a dilemma: some practitioners believed that care plans should be generated through a consultative processes involving patients, while others argued that priority should be placed on generating as many personal care plans as possible, in order to facilitate opportunistic interventions whenever patients came to the health centre and to promote increased monitoring of their conditions through planned recall.

In Katherine West, most personal care plans were assigned by DMOs, with some assigned by RNs, usually, although not always, without formal discussion with the patient concerned. The course taken by the Tiwi Health Board was essentially similar. For both CCTs, formal interviews would have required a considerable amount of time for each client in addition to the substantial time spent creating the plan, and would have been almost impossible to sustain without reduction of other services, or reduced assignment of care plans during the CCT period. These decisions were mainly made by non-Aboriginal practitioners.

In order to discern client awareness of the care plans, the Katherine West evaluation team interviewed a small number $(\mathrm{N}=10$; male and female) of patients living in two neighboring communities, all of whom had been placed on diabetic care plans, in order to gain some sense of these patients' experiences of current clinical practices. A number of themes emerged:

1 All were aware that they had been diagnosed with what most of them called "the sugar" (diabetes).

2 All said that they reported regularly to the clinic to replenish their supply of tablets, which they claimed to take as directed.

3 All reported that, while at the clinic, they were subjected to various "check-ups", mainly blood pressure, urine tests, blood tests. They understood these to be necessary to monitoring their health with respect to "the sugar".

$4 \quad$ All associated diabetes with excessive sugar intake, and reported having been urged by clinic staff to drink less beer and Coca Cola, and to cut out sugar with 
tea. Some had taken the advice to heart. One reported having switched from Coca Cola to a dietary soft drink. Several reported having struggled to reduce smoking, although none claimed to have succeeded in quitting.

5 All spoke positively about the health centre, and several mentioned the positive impact of having a resident doctor for the first time.

6 None, however, knew anything about care plans. None, it was clear, had been involved in planning their own health care.

The Tiwi experience was not dissimilar, although Tiwi patients had had longer experience of CCTIS as a functioning system than their counterparts at Katherine West. A series of two to three day periods of participant observation in health centres indicated that they were not aware of having been drawn into the care planning process nor of their care plans as such. However, most patients indicated an awareness that "the computer" played a role in informing practitioners and patients alike of services due. They were sometimes shown a screen by practitioners, and offered the services shown as due. The patients then would often accept or refuse the offer of service. Although these practices were highly variable across and even within the health centres, a number of non-Tiwi practitioners interviewed thought that patients were indeed developing a greater sense of responsibility for their own choices - rather than simply passively accepting or resisting service demands, or showing poor "compliance". In at least one Tiwi clinic where the evaluators had the opportunity for sustained observation, many patients had at least some discussion, initiated usually by the nurse, sometimes the AHWs, concerning what services were due; they would usually accept, but sometimes postponed services for another appointment. In a separate clinic at the same health centre, almost no conversations of this kind were occurring, and very few services were being delivered opportunistically by AHWs. In other words, patient exposure to the care plans through reference to the computer, and through active encouragement of responsibility for choice was variable.

Where does this leave the Trial planners' expectations that patient "empowerment" would be an outcome of care coordination? A wide gulf exists between the worldviews of Aboriginal clients and the non-Aboriginal practitioners, in terms of both medical and non-medical cultural expectations. The differences between Aboriginal and nonAboriginal understandings also extends to AHWs, despite their experience with the externally derived medical system. Aboriginal clients are on the one hand to some extent passive recipients of health services managed and delivered by non-Aboriginal 'experts', with limited capacity to actively engage with them in informed ways. On the other hand, Aboriginal clients are not simply passive or powerless, but in fact very often make forceful demands on AHWs and non-Aboriginal practitioners to respond to needs in certain ways, demanding some kinds of response while frequently refusing to comply with medical demands, even when conveyed by AHWs. While it may have been unreasonable to expect that care coordination could have greatly transformed these relationships within the three years of the trials to date, it should not be assumed that the CCTs do not have the potential to achieve change in patient engagement with the health care system over the medium term, perhaps through pathways other than care coordination in the narrow sense. 
However, given the imbalance of responsibilities between AHWs and non-Aboriginal practitioners in the delivery of care plan services as detected in the Tiwi CCT, it appears that improved patient self-responsibility and "compliance" will not necessarily be secured by recruitment of AHWs alone, as some recent work has tended to imply (cf. Humphery, K., \& Weeramanthri, T., 2001). Further development of their contribution to care planning and case management in the health centre setting is desirable.

\section{Conclusions and Implications}

The implementation of care coordination was subject to two parallel and inter-related processes. The first involved the consolidation of health centre management within the larger organizational framework of service delivery managed by the Health Boards, with their distinctive charter to achieve outcomes both at the population-wide level and in clinical case management. Secondly, care coordination involved the introduction and development of new work practices in the health centres, with their existing style of relations between clients and practitioners which embodied an orientation to reactive, acute care rather than prevention. The existing style of practice was to some extent resistant to a new "managerialism" represented by the Health Boards and the CCT information infrastructure.

On the Tiwi Islands, the potential to achieve improvements in services according to protocol was demonstrated. However, the outcomes at transition year indicate that there remained unresolved questions about the ability of the Board to sustain the improvement to quality of care through the care coordination model, that is, through continuing improvement in the opportunistic and planned delivery of care plan services. Aspects of the pre-existing local style of service delivery, with its orientation to reactive delivery of acute care in response to the urgent demands of clients, persisted. This reflected some basic continuities in AHW relations with clients as fellow community members and kin, and a distinctive pattern of negotiation of their relations with less permanent nonAboriginal practitioners who mediate links with external managerial frameworks. Both the Tiwi and the Katherine West evaluations indicate that it is as yet unclear to what extent the care planning process will affect the manner in which Aboriginal patients engage with the health care system.

In both trials, the Boards discovered significant gaps in basic infrastructure and resources, the rectification of which consumed considerable resources. For Katherine West, key tasks were the consolidation of appropriate staffing levels, including the replacement of DMOs by resident general practitioners, and the recruitment of additional AHWs. These had only been achieved by late in the transition year. The outcomes of the evaluation indicate that resources and management at health centre level in Katherine West had not reached a threshold at which sustained changes in service delivery according to adopted protocol could have been achieved in all eight health centres within the limited time frame of the CCT. 
Under the old departmental system there was no compelling connection between the managerial needs for strategic information on services and outcomes and the actual organization and delivery of practice according to standards of accountability represented by practice guidelines and their implied clinical objectives. Management had only weak controls and limited capacity to systematically develop strategy for clinical practice, say in treatment and care of the chronically ill. With the establishment of the two Health Boards and the potential of CCTIS and related infrastructure to delivery high quality, clinically relevant strategic information, the possibility of stronger accountability in clinical practice exists. However, the capacity to establish direction in clinical practice is not given with the technologies, but must be built up within local systems of management. It entails development of the roles of all personnel and subtle modification of client expectations through pro-active clinic consultation activity with an emphasis on prevention and health promotion. The capacity to use the information in CCTIS to answer questions about clinical practice is partly shaped by the maturation of the local management of clinical processes. This means addressing issues of participation and responsibility of all practitioners, including Aboriginal practitioners. There should be no need to make the choice between technically improved services which leave the AHWs behind, at the margins, and improvement of services through more active engagement of the AHWs.

The NT Coordinated Care Trials have set in place structural arrangements and processes which can deliver substantial increases in Aboriginal participation in decision-making about health generally and within direct service delivery. Through the development of community-based services, the Boards now employ substantial numbers of community members in a range of activities aimed at prevention and health promotion. The Health Boards' orientation to community development has in turn created the need to address systematically the many complex constraints on Aboriginal participation in employment and education. The CCTs have thereby brought governments, through the tasks taken on by the two Aboriginal Health Boards, to address issues fundamental to health service development. Some of these, like education and social development in the communities, are fundamentally bound up with the social determinants of health improvement among Aborigines in the NT over the longer term.

\section{Acknowledgements}

The research for this paper is a result of local evaluations of two Aboriginal Coordinated Care Trials in the Northern Territory, funded by the Commonwealth Department of Health and Aged Care, Canberra Australia. The authors would like to acknowledge the generous assistance and cooperation of the Katherine West and Tiwi Health Boards, and their staff in the community health centres and programs, management and community members, and the assistance of staff of Territory Health Services, Darwin, and of the Commonwealth Department of Health and Aged Care, Darwin and Canberra. 


\section{References}

Commonwealth Department of Health and Aged Care, 2001, The Aboriginal and Torres Strait Islander Coordinated Care Trials: National Evaluation Summary, Canberra, AGPS, March.

D’Abbs, P., Togni, S., Bailie, R., Fitz, J., Wales, N., 2001: "Katherine West Coordinated Care Trial Transition Year (April 2000 - March 2001) Evaluation Report”, Darwin, MSHR.

Humhery, K., Weeramanthri, T., 2001, Forgetting Compliance: Aboriginal health and medical culture, Darwin, CRCATH \& NTU Press.

Local Evaluation Team, 2000, Jirntangku Miyrta Katherine West Coordinated

Care Trial Local Evaluation Final Report, Menzies School of Health

Research, Darwin.

McDermott, R., "Improving Equity and Efficiency in the Bush: a Needs-Based Method for Health Care Resource Allocation in Remote Communities", AUST J. RURAL HEALTH, 3, 1995, pp. $72-79$.

McClintock, C., 1990, "Evaluators as applied theorists", EVALUATION PRACTICE, 11(1), 1-12.

McLennan, W., \& Madden, R., 1999, The Health of Australia's Aboriginal and Torres Strait Islander Peoples, Canberra, Australian Bureau of Statistics.

Pawson, R., \& Tilley, N., 1997, Realistic Evaluation, London, Sage.

Robinson, G., \& Bailie, R., 2000, Tiwi Coordinated Care Trial Final Local evaluation Report, Darwin, Northern Territory University.

Robinson, G., Bailie, R., Togni, S. \& Kondalsamy-Chennakesavan, S., 2001, Tiwi

Coordinated Care Trial Transition Year Evaluation Report, Darwin, Northern Territory University.

Robinson, G., \& Bailie, R., Tiwi Coordinated Care Trial Final Local Evaluation Report, Darwin, Northern Territory University, 2000.

\section{Authors' Details:}

Dr Gary Robinson, Director, Centre for Social Research, Northern Territory University, Darwin, Northern Territory, Australia.

Postal:

Centre for Social Research, NTU, 
Darwin, NT, 0909,

Australia.

Gary Robinson has taught and carried out research in Psychological Anthropology and Sociology in Darwin since 1985. His special interests have included Aboriginal adolescence, family life, and social problems including violence, suicide and self harm among youth. Since 1997 he has been engaged in the evaluation of health service developments including the Tiwi Coordinated Care Trial and is currently engaged in research into management of services to the chronically ill and the evaluation of early intervention initiatives to reduce the incidence of self harm and suicide among remote indigenous youth.

Dr Peter d'Abbs, Senior Lecturer, Menzies School of Health Research, Darwin, Northern Territory, Australia, and Queensland Health, Cairns, Queensland, Australia.

Postal:

Northern Zone Management Unit, Queensland Health,

PO Box 1103, Cairns, Qld., Australia 4870

Peter d'Abbs has exensive experience in conducting health and social policy related research, especially in rural and remote settings. Major projects in recent years have involved evaluations of community-based alcohol interventions in several regional towns, preparation of regional alcohol strategies for community groups, and having principal responsibility for designing and conducting the evaluation of the Katherine West Coordinated Care Trial. He currently holds a joint appointment, as a Senior Lecturer at the Menzies School of Health Research in Darwin, Australia, and as Alcohol and Other Drugs Adviser with Queensland Health, Cairns, Australia. In the latter capacity he is engaged in developing an evidence-based model for the provision of alcohol and other drug services in rural and remote regions.

Dr Ross Bailie, Associate Professor of Public Health, Menzies School of Health Research and Flinders University NT Clinical School. MBChB, MPhil (Maternal and Child Health), MD (Community Health), FAFPHM.

Postal:

Menzies School of Health Research,

PO Box 41096,

Casuarina, NT 0811,

Australia.

Ross Bailie has worked in general practice and hospital practice for 7 years before training in public health in South Africa. Research work over the past 10 years has been strongly oriented to health services and health policy issues, predominantly in

disadvantaged communities in South Africa and Australia. Current research

interests are health related infrastructure and health services.

Samantha Togni, Senior Research Officer, Menzies School of Health Research, Darwin, Northern Territory, Australia.

Postal:

Menzies School of Health Research,

PO Box 41096,

Casuarina, NT 0811,

Australia. 
Samantha Togni has a Master of Arts by research from the University of Melbourne and over the last seven years has worked as a social researcher based in Darwin, working with the Menzies School of Health Research on several evaluations of community initiatives relating to alcohol problems. Since mid-1998 Samantha was the Project Manager/Senior Research Officer in the Local Evaluation Team which evaluated the Katherine West Coordinated Care Trial. She is engaged in ongoing research with the Katherine West Health Board and teaches Sociology and Health as part of a Masters of Public Health course. 